\title{
The Low-Q Deep-Inelastic Scattering Data in the Global Fit of PDFs
}

\author{
S. Alekhin ${ }^{1}$, S. Kulagin ${ }^{2}$, R. Petti ${ }^{3}{ }^{*}$ \\ 1- Institute for High Energy Physics \\ 142281 Protvino, Moscow region - Russia \\ 2- Institute for Nuclear research \\ 117312 Moscow - Russia \\ 3- South Carolina University - Department of Physics and Astronomy \\ Columbia SC 29208 - USA
}

\begin{abstract}
We check impact of the existing deep-inelastic scattering (DIS) data at low momentum transfer $Q$ on the global QCD fit of parton distribution functions (PDFs). We find that DIS data are described well within the NNLO QCD approximation with the target mass and phenomenological high-twist corrections down to $Q \sim 1 \mathrm{GeV}$. The twist-4 terms in the DIS structure functions are extracted; for $F_{\mathrm{L}}$ its value is comparable to 0 within the errors at $x \gtrsim 0.1$. The updated PDF set with reduced uncertainties and relevant for modeling the low- $Q$ lepton-nucleon scattering is obtained.
\end{abstract}

The existing DIS data at small momentum transfer $Q$ in principle can put valuable constraints on the parton distribution functions (PDFs) and the strong coupling constant $\alpha_{\mathrm{s}}$ due to their very good statistical precision. Besides, the low- $Q$ DIS data are necessary for clarification of the limits at which the parton model is valid. A study of this kinematical region in terms of the perturbative QCD is labored due to significant high-order QCD corrections, however recent progress in the NNLO QCD calculations [2] allows to use the DIS data down to $Q \sim 1 \mathrm{GeV}$ for a fit of PDFs keeping perturbative stability under control. The power corrections to the perturbative QCD terms should be also taken into account. These corrections appearing in the formalism of operator-product expansion (OPE) spoil factorization therefore one has to consider its impact on the PDFs extracted, particularly in analysis of the low- $Q$ data. In this communication we report our results on using the DIS data down to $Q=1 \mathrm{GeV}$ in the global QCD fit of PDFs with the power corrections due to the kinematical target mass effects [3] and the dynamical high-twist (HT) terms included into analysis.

The analyzed data set consist of the world charged-leptons DIS cross section data for the proton and deuteron targets by the SLAC, BCDMS, NMC, FNAL-E-665, H1, and ZEUS experiments supplemented by the fixed-target Drell-Yan data, the latter constrain the sea quark distribution, which is poorly determined by the DIS data alone. Basically the same combination of data was used in the earlier fit of Ref.[4] with the cut $Q^{2}>2.5 \mathrm{GeV}^{2}$ imposed on the DIS data. In the present fit alongside with the softer cut imposed on the SLAC and NMC data, $Q>1 \mathrm{GeV}$, we also add the DIS data by FNAL-E-665 experiment [5] since they give additional constraint on the PDFs at small $x$ provided not too stringent cut on $Q$ is applied. The cut on invariant mass of the hadron system $W>1.8 \mathrm{GeV}$ is imposed on the DIS data to avoid resonance region. The total number of data points (NDP) used in the fit is 3076 , in the range of $x=0.0001 \div 0.9$. The analysis is performed in the NNLO QCD approximation with the target mass corrections taken into account, the dynamical twist- 4

${ }^{*}$ This work is partially supported by by the RFBR grant 06-02-16659 and the Russian Ministry of Science and Education grant Nsh 5911.2006. 
terms parameterized in the additive form as

$$
F_{2, T}\left(x, Q^{2}\right)=F_{2, T}^{\mathrm{twist}-2}\left(x, Q^{2}\right)+\frac{H_{2, T}(x)}{Q^{2}\left[\mathrm{GeV}^{2}\right]},
$$

and correction for the nuclear effects in the form of model [6] applied to the deuteron data $^{a}$.

As a first step of the analysis we checked possible twist-6 contribution to the DIS structure functions (SFs) adding the terms $H^{(6)}(x) / Q^{2}$ to Eq.(1). Since the twist-6 terms are insensitive to the large- $x$ part of the data used due to the cut on $W$ they were set to 0 at $x \geq 0.5$. Values of the HT terms at $x=0$ were also set to 0 in view of no clear evidence of the saturation effects were found at the small $x$ HERA data. The HT terms in the SFs $F_{2, \mathrm{~T}}$ obtained in this variant of the fit are given in Fig.1. Surprisingly, we observe big positive contribution of the twist- 6 term to $F_{\mathrm{T}}$ at $x \sim 0.15$. At the same time this contribution is compensated by the negative twist- 4 contribution. Because the twist- 4 and twist- 6 coefficients are similar in magnitude and opposite in sign, the sum of these terms demonstrate weak dependence on $Q$. This leads us to the con-

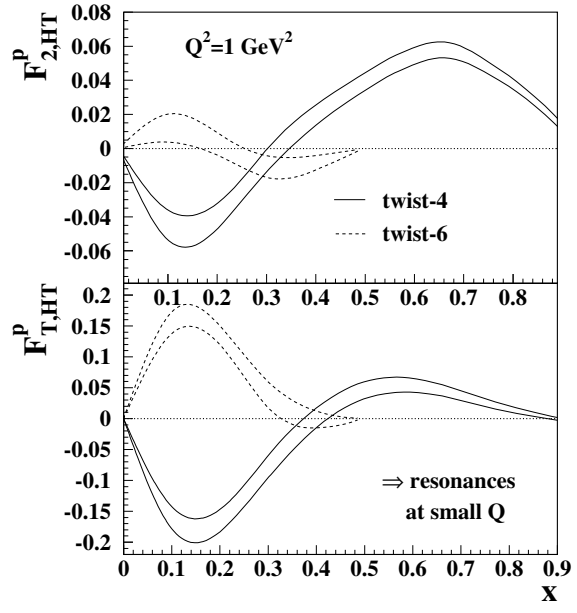

Figure 1: The $1 \sigma$ error bands for the twist4 (solid lines) and twist-6 (dashes) terms in the proton $F_{2}$ (upper panel) and $F_{\mathrm{T}}$ (lower panel). The arrow indicates region of $x$ with the limited potential for determination of the twist-6 terms due to the cut on $W$. clusion that in fact the twist- 6 term in $F_{\mathrm{T}}$ absorbs some non-power-like effects. Indeed, in the variant of fit with no twist- 6 terms included magnitude of the pulls is maximal not at the smallest $Q$, but at $Q^{2} \sim 7 \mathrm{GeV}^{2}$, in the region of overlapping of the SLAC and BCDMS data (see Fig.2). These two data sets demonstrate certain discrepancy, which goes into the fake twist- 6 contribution, if this term is fitted too. The values of inelasticity $y$ are big for the SLAC data at largest $Q$ and small for the BCDMS data at lowest $Q$. For this reason the discrepancy affects mostly the HT terms in $F_{\mathrm{T}}$, which is more sensitive to $y$ than $F_{2}$. At the same time the SLAC/BCDMS data discrepancy at $Q^{2} \sim 7 \mathrm{GeV}^{2}$ is not crucial for determination of the twist-4 terms. If we rescale the errors in data at this region to bring the pulls to the level of $1 \sigma$ the corresponding increase of the HTs errors is insignificant. Evidently, this terms are driven by the data at lowest $Q$ used and in principle the SLAC/BCDMS data around $Q^{2} \sim 7 \mathrm{GeV}^{2}$ can be even dropped without the loss of statistical sensitivity of the total data sample. Preliminary data by the experiment JLAB-E-118 [7] are in agreement to the SLAC data at low $Q$, this is in favor of reliability of the latter at $Q \sim 1 \mathrm{GeV}$, despite their possible defects in the region of overlapping with the BCDMS data.

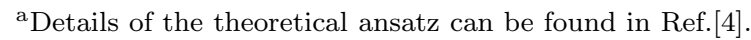


Coming to the conclusion that the twist6 terms observed are just due to certain discrepancy in the data we drop these terms in the final version of the fit. The value of $\chi^{2} / \mathrm{NDP}$ for this variant of fit is $3815 / 3076=1.25$, bigger than 1 particularly due to certain discrepancies in data discussed above. Nonetheless the inconsistent data points are spread more or less randomly over kinematics and do not bias results of the fit; rescaling of the errors in the data points with the biggest pulls, which brings the overall value of $\chi^{2} / \mathrm{NDP}$ to 1 leads to increase of errors in the PDFs and HTs within $20 \%$ only. The twist- 4 terms obtained in final version of our fit are given in Fig.3. The HT terms in $F_{T}$ are remarkably similar to ones in $F_{2}$, despite these two terms were fitted independently. As a result the HT term in $F_{L}$ defined as $H_{\mathrm{L}}=H_{2}-H_{\mathrm{T}}$ is well comparable to 0 within the errors. The HT contribution to the structure function $R=\sigma_{\mathrm{L}} / \sigma_{\mathrm{T}}$ is also small in the whole range of $x$ considered. This result is different from the conclusion of Ref.[8] about big HT contribution to $R$. We explain this difference by the fact that the low- $Q$ part of the SLAC data was not considered in Ref.[8], extrapolation of those results to the lower $Q$ must be in disagreement to the data. The total contribution of the HT terms into the DIS cross section is small as compared to the leading-twist (LT) part. For the realistic DIS kinematics the ratio of the HT and LT terms is $\lesssim 10 \%$ that justifies the use of twist expansion in our analysis.

The value of $\alpha_{\mathrm{s}}$ is similar to one obtained in Ref.[4] with the cut $Q^{2}>2.5 \mathrm{GeV}^{2}$ and is in good agreement to the result of the nonsinglet DIS data analysis [9]. The PDFs obtained in the fit with the low- $Q$ DIS data included are also not very different from ones of Ref.[4]. This manifests good separation of the LT and HT terms in the fit, correlation coefficients for the LT and HT terms do not exceed 0.3 for the whole range of $x$. The most significant change is in the gluon distribution at $x \sim 0.3$, which goes up in the low- $Q$ fit (see Fig.4). This is because of significant twist-4 term in $R$ appearing in a fit with the cut $Q^{2}>2.5 \mathrm{GeV}^{2}$, similarly to the analysis of Ref.[8], the LT in $R$ is suppressed, correspondingly. Since the LT in $R$

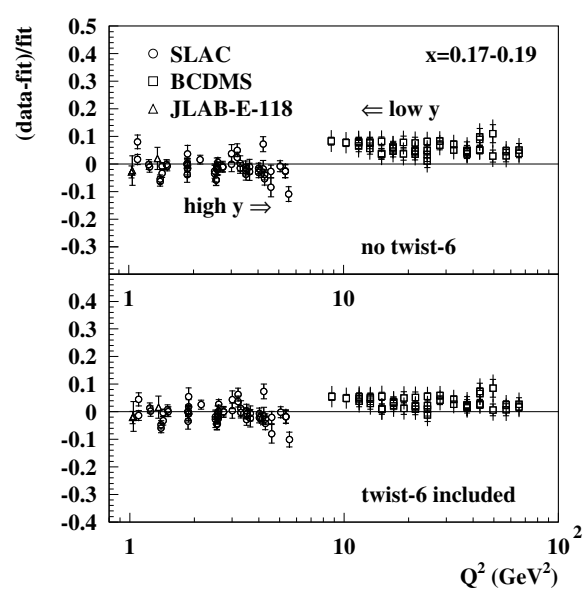

Figure 2: The pulls for the variants of the fit with and without twist- 6 terms taken into account. The arrows in the upper panel indicate the high- $y$ and low- $y$ regions for the SLAC and BCDMS data. The data points for JLAB-E118 experiment at $Q \sim 1 \mathrm{GeV}$ were not used in the fit. 
is proportional to the value of gluon distribution the latter is suppressed too, as a result.

The errors in PDFs are improved as compared to the fit of Ref.[4], in particular the $d$-quark distribution is now determined with the precision of several per cent at $x \sim 0.2$, comparable to precision of the $u$-quark distribution. This improvement has important phenomenological impact on extraction of the Weinberg angle from the neutrino data. For the analysis by $\mathrm{NuTeV}$ collaboration [10] based on the Paschos-Wolfenstein relation a good knowledge of the valence distributions in this region is required to guarantee accurate estimation of the correction for the target nonisoscalarity [11]. This correction is proportional to the ratio $x_{1} / x_{0}$, where $x_{1}$ and $x_{0}$

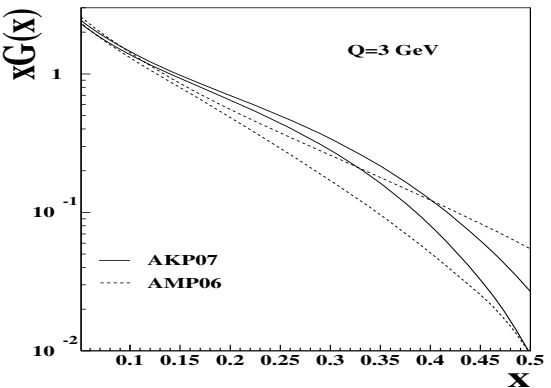

Figure 4: The $1 \sigma$ error bands for the gluon distribution obtained in our fit (solid lines) compared to one of Ref.[4](dashes). are the integrals over $x$ of the iso-vector and iso-scalar combinations of the valence quarks, respectively. For the PDFs obtained in our fit we get $x_{1} / x_{0}=0.424(6)$ that provides uncertainty in the target non-isoscalarity correction comparable to the experimental errors in the $\mathrm{NuTeV}$ data.

In summary, we conclude that existing DIS data can be well described down to $Q \sim$ $1 \mathrm{GeV}$ within the NNLO QCD approximation combined with the phenomenological hightwist corrections. The twist- 4 terms in $F_{2, T}$ extracted from the global QCD fit to the DIS data supplemented by the fixed-target Drell-Yan data are found to be within $10 \%$ of the LT terms for the kinematics considered, while the HT contribution to $F_{L}$ is found to be comparable to 0 within the errors at $x \gtrsim 0.1$. Obtained PDFs set with improved precision and tuned to the low- $Q$ DIS data can be used for modeling the low- $Q$ leptonnucleon scattering.

\section{References}

[1] Slides: http://indico. cern. ch/contributionDisplay $\cdot$ py? contribId=29\&sessionId=8\&conf Id=9499

[2] S. Moch, J. A. M. Vermaseren and A. Vogt, Nucl. Phys. B 688 (2004) 101; A. Vogt, S. Moch and J. A. M. Vermaseren, Nucl. Phys. B 691 (2004) 129; J. A. M. Vermaseren, A. Vogt and S. Moch, Nucl. Phys. B 724 (2005) 3; S. Moch and M. Rogal, arXiv:0704.1740 [hep-ph].

[3] O. Nachtmann, Nucl. Phys. B 63 (1973) 237; H. Georgi and H. D. Politzer, Phys. Rev. D 14 (1976) 1829.

[4] S. Alekhin, K. Melnikov and F. Petriello, Phys. Rev. D 74 (2006) 054033.

[5] M. R. Adams et al. [E665 Collaboration], Phys. Rev. D 54 (1996) 3006.

[6] S. A. Kulagin and R. Petti, Nucl. Phys. A 765, 126 (2006).

[7] C. Keppel, private communication.

[8] J. L. Miramontes, M. A. Miramontes and J. Sanchez Guillen, Phys. Rev. D 40 (1989) 2184.

[9] J. Blümlein, H. Bottcher and A. Guffanti, Nucl. Phys. B 774 (2007) 182.

[10] G. P. Zeller et al. [NuTeV Collaboration], Phys. Rev. Lett. 88 (2002) 091802 [Erratum-ibid. 90 (2003) 239902].

[11] S. A. Kulagin, Phys. Rev. D 67 (2003) 091301. 